\title{
Attitude Optimization Control of Unmanned Helicopter in Coal Mine Using Membrane Computing
}

\author{
Jiachang Xu $\mathbb{D}^{1}{ }^{1}$ Yourui Huang $\mathbb{D}^{2},{ }^{2}$ and Yu Liu ${ }^{3}$ \\ ${ }^{1}$ School of Computer Science and Engineering, Anhui University of Science and Technology, Huainan 232001, China \\ ${ }^{2}$ School of Electrical and Information Engineering, Anhui University of Science and Technology, Huainan 232001, China \\ ${ }^{3}$ State Key Laboratory of Mining Response and Disaster Prevention and Control in Deep Coal Mines, \\ Anhui University of Science and Technology, Huainan 232001, China \\ Correspondence should be addressed to Yourui Huang; hyr628@163.com
}

Received 15 November 2019; Revised 8 March 2020; Accepted 18 March 2020; Published 11 May 2020

Academic Editor: Xuping Zhang

Copyright (C) 2020 Jiachang Xu et al. This is an open access article distributed under the Creative Commons Attribution License, which permits unrestricted use, distribution, and reproduction in any medium, provided the original work is properly cited.

\begin{abstract}
Unmanned helicopter for mission inspection has good application value in intelligent coal mining, and attitude control is important. In this paper, membrane computing is introduced to realize attitude optimization control of an unmanned helicopter. First, we give the application scenarios of unmanned helicopters in coal mines. Secondly, we establish a dynamic model of an unmanned helicopter with environmental participation, and the attitude model of the helicopter is deduced based on this model. Further, the cellular membrane system suitable for the attitude model of an unmanned helicopter under the control parameters of environment mapping is constructed, and the cellular membrane controller based on the characteristics and operation rules of the membrane system is designed. The robust performance of the controller is proved theoretically, and by the semiphysical experiments, the performance of trajectory tracking is almost consistent and attitude angle control is less than $\pm 1^{\circ}$, in the range of $\pm 2^{\circ}$ by wind disturbance. Compared with the linear feedback controller in the same experimental environment, the performance of the membrane controller is improved by nearly 0.4026 on average. It shows that the cellular membrane controller constructed has good effectiveness and robustness. This will provide a good application value for membrane computing in the field of accurate coal mining.
\end{abstract}

\section{Introduction}

Fully reflecting the integration of information, automation, and intelligence, forming a highly intelligent, unmanned, safe, and efficient coal mining way, and truly realizing the goal of intelligent, automated, and unmanned coal safe, efficient, and accurate mining are important [1]. The deep integration of the sensor network, intelligent control, and communication technology and the combination of coal mining demand and underground complex environment are used to realize the whole process monitoring of a coal mine, do a good job in data collection, processing, and feedback real-time accuracy, solve the nonreal time and uncertainty of traditional coal mining disaster early warning, and truly achieve the goal of accurate coal mine disaster early warning difficult problems [2]. Unmanned helicopters with vertical take-off and landing, flexible flight direction, low speed cruise, and other superior flight characteristics, in the military and civilian areas, have shown a good value in the application. Through unmanned helicopters, real-time monitoring, task inspection, investigation, and rescue of coal mines can be completed, so that accurate warning and risk judgment of coal mining disasters can be improved and the new trend of intelligent and accurate coal mining can be deeply integrated. However, the unmanned helicopter itself has strong coupling and underactuated nonlinear characteristics. In the complex underground environment of the coal mine, how to make the unmanned helicopter complete the inspection task under flight control and further improve the application performance of the unmanned helicopter in the coal mine have important theoretical research significance and application value. 
Aiming at the characteristics of the underactuated system of an unmanned helicopter, especially in the aspect of controller design and optimization, domestic and foreign scholars and scientific research institutions have done a lot of theoretical research and practical exploration, and many relevant control theories and methods proposed have been applied and good research results have been obtained. For example, traditional control methods such as PID control [3], $H_{\infty}$ control [4], and LQR control [5, 6] have been proposed and applied successively. The authors in $[7,8]$ used the adaptive method to improve the helicopter attitude control. Oktay et al. [9] proposed a stochastic optimization method to improve the autonomous performance of the helicopter extent. The attitude control and position tracking of an unmanned helicopter by nonlinear control algorithms have been studied in $[10,11]$. The authors in $[12,13]$ used backstepping control theory, neural network [14, 15], and reinforcement learning $[16,17]$ to design the attitude controller of an unmanned helicopter. The constraint model predictive control through physical modeling was done in [18]. The authors in [19-21] achieved good results in improving the helicopter flight performance through power consumption, cost, and other aspects. The above control algorithms and control strategies are concentrated on linear and nonlinear control and intelligent control [22, 23], and both have good performance in terms of control performance and control effect. However, there is still room for improvement in terms of uncertain control stability or external disturbances, especially in the complex environment of the coal mine; in view of the complex dynamic characteristics of the unmanned helicopter, it is necessary to establish an accurate mathematical model suitable for the underground environment, build a controller model suitable for the underground environment of the coal mine, and apply an effective matching algorithm. It is a challenging research topic.

Membrane computing is inspired by the structure and function of biological cells. It is a new branch of natural computing. It abstracts the structure and function of cells into formal processes and fuses each process. Therefore, a new computing mode under membrane computing is generated. Membrane computing was proposed by Păun in 1998, who is the member of the Romanian Academy of Sciences and the European Academy of Sciences [24]. Membrane computing is closely built around the construction of a membrane computing model, which is compared with the standard model. In terms of theoretical research, it analyzes the generality of calculation and compares the calculation efficiency and calculation ability of the model. In the aspect of application research, practical application problems are solved by applying a membrane algorithm [25]. The cellular membrane system model has a clear structure, good hierarchy, and feature distributed and parallel computing. The collection and operation (evolution) rules of the representation objects are flexible, and the membrane algorithm under the system has powerful optimization capabilities; with the universality of Turing machines and the efficiency of computing power, it has its own advantages in the field of optimal control.
In this paper, the optimal control of the helicopter attitude is achieved by better using membrane computing. The main contributions are as follows:

(1) The unmanned helicopter is applied to coal mines for mission inspection.

(2) Analyzing the complex environment under the coal mines that is used as an application scenario to implement helicopter attitude modeling.

(3) Combining the motion characteristics of the helicopter, the cellular membrane system is constructed and the attitude membrane controller is designed. Through theoretical analysis and experimental verification, the feasibility, effectiveness, and stability of the cellular membrane system for helicopter attitude control are proved.

(4) Membrane computing has been expanded in theoretical research, and it has provided new ideas for the precise mining engineering practice of coal mines.

The rest of this paper is organized as follows: Section 2 describes the application scenarios of unmanned helicopters in the coal mine. The method and modeling of unmanned helicopter attitude control under membrane computing are described in Section 3, and the stability analysis is given in theory. In Section 4, the feasibility of the membrane controller is verified through an experimental platform, and it was compared with the linear feedback control to further prove the effectiveness and stability of the membrane controller. Finally, in Section 5, conclusion and future work are presented.

\section{Application Scenario of an Unmanned Helicopter in Coal Mines}

Unmanned helicopter has a wide range of applications and can be applied to agriculture, detection, power inspection, environmental testing, road inspection, and other fields. However, in the coal mines, the application is in infancy. After all, the high level of intelligence required for coal mining is the prerequisite for intelligent coal mining. Robots have played an important role [26]. However, realizing the coal mines' mission inspection with dust, humidity, and wind, an unmanned helicopter has more advantages than a robot, especially in the aftermath of a coal mine disaster, the search and rescue capabilities of a robot are at least far worse than a helicopter. Therefore, the application of a helicopter in coal mines has good development prospects, and the specific application scenario is shown in Figure 1.

The structure of a coal mine can be divided into a roadway model and a working face model; the working environment of unmanned helicopters engaged in mission inspection is divided into normal and postdisaster environments, and the postdisaster working environment changes randomly. Therefore, the unmanned helicopter's coal mine inspection process goes through three main stages: underground environment representation, identification, and decision-making. The specific characteristics are as follows: 


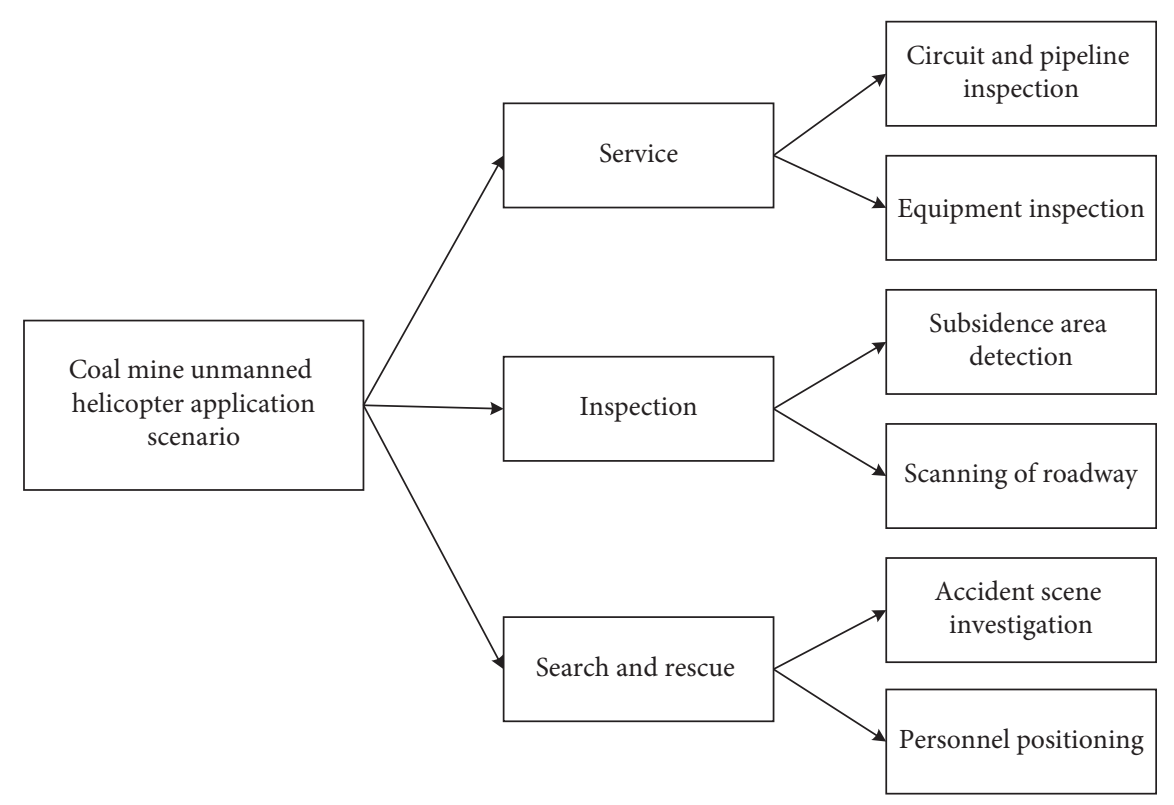

Figure 1: Application scenario of an unmanned helicopter in coal mines.

(1) Constructing a contextual representation based on the characteristics of the coal mine system.

(2) Based on the perception of the environment by an unmanned helicopter, this paper uses a lidar and depth camera and the depth camera includes inertial sensors.

(3) Constructing the behavior model of an unmanned helicopter based on the environment perception of (2).

(4) The helicopter's own decision depends on the mapping of (2) and (3).

(5) Controlling own behavior based on the dynamics of unmanned helicopters.

It is that the establishment of a mathematical model of the underground environment of coal mines is the basis for autonomous decision-making of unmanned helicopters. While satisfying the above characteristics, it should also have the following:

(l) Real-time scenes of the coal mines.

(2) Data (parameters) collection, processing, and analysis.

(3) Data (parameters) should have valid characteristic values.

(4) Modeling a dependent environment for helicopter behavior decision.

(5) The environmental model reflects the accuracy of the unmanned helicopter behavior.

Unmanned helicopters must have their own recognition and perception of the flying environment when flying under the coal mine, whether it is the inspection under normal circumstances or search and rescue after the disaster, and the helicopter itself is relied upon to complete the environmental modeling process, including data collection and processing. The designed membrane algorithm and necessary airborne computers and various sensors such as lidar and depth camera are utilized, and the specific equipment and parameters are shown in Table 1. Therefore, only the unmanned helicopter modeling in line with the coal mine environment can ensure the flight performance and safety of the helicopter and better complete the various mission inspection of the unmanned helicopter.

\section{Method and Modeling of Attitude Optimization Control}

3.1. Attitude Dynamics Model. Establishing the correct coordinate system and the conversion relationship between the coordinate systems are the premises of characterizing the movement state of the unmanned helicopter. To achieve attitude control of an unmanned helicopter in a nonlinear system, the attitude angle needs to be controlled, that is, three Euler angles $(\varphi, \theta$, and $\psi)$ relative to the ground coordinate system $\left(\mathrm{O}-\mathrm{X}_{\mathrm{E}} \mathrm{Y}_{\mathrm{E}} \mathrm{Z}_{\mathrm{E}}\right)$. To achieve the purpose of controlling the tilt angle and the deflection angle of the unmanned helicopter relative to the ground coordinate system, it is necessary to ensure the correctness of the conversion relationship between the body coordinate system (O-XYZ) and the ground coordinate system, as shown in Figure 2 [27].

Each of the three Euler angle rotates around an axis, that is, the corresponding pitch angle $\theta$, roll angle $\varphi$, and yaw angle $\psi$. In Figure 2, the projection of the rotational angular velocity on the body axis is $p, q$, and $r$ and the projection of the helicopter velocity on the body axis is $u, v$, and $w$. Marks $v$ and $w$ represent the coordinate vectors of linear velocity and angular velocity in the ground coordinate system, respectively, and the vectors of $w$ in the body coordinate system are represented as [28] 
TABLE 1: Main hardware information parameters of the experimental platform.

\begin{tabular}{lc}
\hline Device & Parameter information \\
\hline Helicopter stand & JCZK450 L \\
Development board & NVIDIA Jetson TX2 \\
Inertial measurement unit & MTi-8ATG6 \\
Depth camera & Intel RealSense D435i \\
Motor & HF500 \\
\hline
\end{tabular}

$$
\begin{aligned}
& v=i_{B} u+v_{B}+k_{B} w, \\
& \omega=i p+j q+k r,
\end{aligned}
$$

where $u$ is the $O X$ axis speed, $v$ is the $O Y$ axis speed, $w$ is the axis speed, $p$ is the $O X$ axis angular velocity, $q$ is the $O Y$ axis angular velocity, and $r$ is the $O Z$ axis angular velocity.

$$
\text { By rotating the matrix } R=
$$
$\left[\begin{array}{ccc}c_{\varphi} c_{\theta} & -c_{\varphi} s_{\theta}+c_{\varphi} s_{\varphi} s_{\theta} & s_{\varphi} s_{\phi}+c_{\varphi} s_{\theta} c_{\phi} \\ s_{\varphi} c_{\theta} & c_{\phi} c_{\varphi}+c_{\varphi} s_{\phi} s_{\theta} & c_{\phi} s_{\varphi}+s_{\varphi} s_{\theta} c_{\phi} \\ -s_{\theta} & c_{\theta} s_{\phi} & c_{\phi} c_{\theta}\end{array}\right], s_{t}$ and $c_{t}$ stand for trig functions $\sin (t)$ and $\cos (t), t \in\left[\begin{array}{lll}\varphi & \theta & \phi\end{array}\right]$. In the ground coordinate system, the position, attitude, and translation motion of the helicopter are inferred; marking the helicopter center of gravity position $\dot{p}=\left[p_{x}, p_{y}, p_{z}\right]$, the dynamic equations of the helicopter position and speed in the ground coordinate system can be obtained:

$$
\begin{aligned}
& \dot{p}=v, \\
& \dot{v}=\frac{f_{B}}{m} R,
\end{aligned}
$$

where $f_{B}$ is the external force and $R \in S O$ (3). Combined with the derivative of the rotation matrix, we can get

$$
\begin{aligned}
\dot{R} & =\breve{\omega}_{B} R, \\
\dot{\omega}_{B} I & =\tau_{B}-\omega_{B} \times\left(\omega_{B} I\right),
\end{aligned}
$$

where $\omega_{B}=\left[\begin{array}{lll}p & q & r\end{array}\right]^{T}$ and $I$ is the inertia matrix. To simplify the design strategy and ignore the influence of tail thrust, the helicopter is affected by the main rotor and gravity, and the mechanical vector is

$$
f_{B}=R^{T}\left[\begin{array}{l}
0 \\
0 \\
G
\end{array}\right]+\left[\begin{array}{c}
0 \\
0 \\
-T_{M}
\end{array}\right] .
$$

The torque vector generated by the force and rotor moment in formula (7) is

$$
\tau_{B}=\left[\begin{array}{c}
y_{m} Z_{M}-z_{M} Y_{M}-z_{t} Y_{T} \\
z_{m} X_{M}-x_{m} Z_{M} \\
x_{m} Y_{M}-y_{m} X_{M}+x_{t} Y_{T}
\end{array}\right]+\tau_{Q},
$$

where $\left[x_{m}, y_{m}, z_{m}\right]$ is the main rotor position vector and $\left[x_{t}, y_{t}, z_{t}\right]$ is the tail rotor position vector. From formulas (7) and (8), the helicopter attitude dynamics equation is as follows:

$$
\begin{aligned}
I \dot{\omega}_{B}= & -\omega_{B} \times\left(I \omega_{B}\right)+\left[\begin{array}{ccc}
-Q_{M}\left(T_{M}\right) & -z_{m} T_{M}+k_{\beta} & z_{t} \\
-z_{m} T_{M}+k_{\beta} & Q_{M}\left(T_{M}\right) & 00 \\
y_{m} T_{M} & x_{m} T_{M} & -x_{t}
\end{array}\right] v_{c} \\
& +\left[\begin{array}{c}
-y_{m} T_{M} \\
x_{m} T_{M} \\
-Q_{M}\left(T_{M}\right)
\end{array}\right],
\end{aligned}
$$

where $Q_{M}$ is the reaction moment of the rotor and $T_{M}$ is the rotor thrust vector.

3.2. Numerical Cell Membrane System Design. The cellular membrane system is a kind of numerical membrane systems with a layered membrane structure and a distributed parallel computing model [29]. Considering the characteristics of unmanned helicopter parameters, the definition degree is $m(m \geq 1)$.

$\prod=\left\{m, H, \mu,\left[\operatorname{Var}_{1}, \operatorname{Pr}_{1}, \operatorname{Var}_{i}(0)\right], \ldots,\left[\operatorname{Var}_{m}, \operatorname{Pr}_{m}, \operatorname{Var}_{m}(0)\right]\right\}$,

where

(1) $H$ is the membrane marker set;

(2) $\mu$ is the membrane structure with the inclusion degree of $m$;

(3) $\operatorname{Var}_{i}$ is a set of variables in the membrane structure region, $1 \leq i \leq m$;

(4) $\operatorname{Var}_{i}(0)$ is the initial value of variable $\operatorname{Var}_{i}$;

(5) $\mathrm{Pr}_{i}$ is a set of rules in the membrane structure area, including the generation rules and distribution rules of values. Rule $t$ is defined as

$\operatorname{pr}_{t, i}=\left[f_{t, i}\left(x_{1, i}, \ldots, x_{k_{i}, i}\right), \lambda_{t, 1}\left|v_{1}+\lambda_{t, 2}\right| v_{2}+\ldots+\lambda_{t, n_{i}} \mid v_{n_{i}}\right]$.

In formula (11), $f_{t, i}\left(x_{1, i}, \ldots, x_{k_{i}, i}\right)$ is the generation rule of value, $n_{i}$ is the number of variables in the allocation rule of participating values in membrane $i, k_{i}$ is the number of variables executed by the generation rule of all values in membrane $i, v_{1} \sim v_{n}$ is the variable distribution object, $\lambda_{1} \sim \lambda_{n}$ is the distribution proportion coefficient of value, and $\lambda_{t, 1} \mid v_{1}+$ $\lambda_{t, 2}\left|v_{2}+\ldots+\lambda_{t, n_{i}}\right| v_{n_{i}}$ is the distribution rule of the value. When the value generation rule calculates the value of $f$, dividing by the sum of the distribution proportion coefficient $\lambda_{1} \sim \lambda_{n}$ of the value, $\lambda_{1} \sim \lambda_{n}$ calculates the proportion assigned to variable $v_{1} \sim v_{n}$ for a cycle. If there are the same variables in the calculation process in the value allocation process of the membrane, the calculated value of the membrane is not allocated to each variable, and the final value of the variable is the cumulative value of the calculation. If there is a value of the variable in the generation rule, after the implementation of the value of the allocation rule, the value is reset as 0 and then the initial state value is further summed with the allocated value to get a new value. 


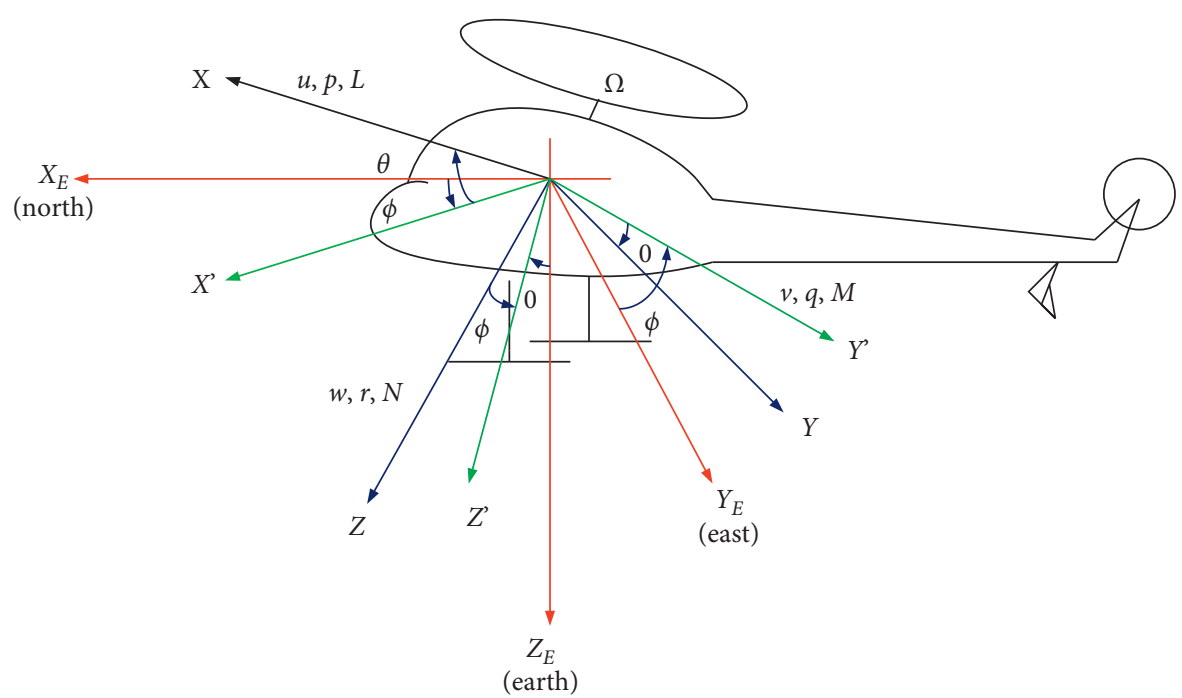

FIgURE 2: Relationship between the airframe coordinate system and the ground coordinate system.

3.3. Membrane Controller Design. In view of the superior parallel computing ability of membrane computing, considering the four channel and dynamic tracking characteristics of the helicopter, the attitude membrane controller system as shown in Figure 3 is established, which aims to establish the membrane controller for experimental simulation later, interpret the membrane controller and parallel computing of the data, and output the results to the execution unit.

According to formula (12) and the process of building a helicopter dynamics model (3)-(9), considering the structural characteristics of the membrane system and the flexibility of communication rules, the control rate rules are executed in sequence, setting a complete calculation cycle of a membrane controller which needs 4 cycles to complete, and the final calculation results can be obtained through the cycle execution. The construction of an unmanned helicopter attitude membrane controller is shown in Figure 4.

According to formula (5), if $e_{\varphi}$ is the yaw angle error, the dynamics of the yaw angle error is

$$
\dot{e}_{\varphi}=\frac{s_{\phi}}{c_{\theta}} q+\frac{c_{\phi}}{c_{\theta}} r-\varphi_{r}
$$

where $\varphi_{r}$ represents the yaw dynamics and $r$ is the yaw rate pseudocontrol; the error dynamics of the corresponding yaw angle is

$$
\dot{e}_{\varphi}=\frac{s_{\phi}}{c_{\theta}} q+\frac{c_{\phi}}{c_{\theta}} r_{d}-\varphi_{r}+\left[0,0, \frac{c_{\phi}}{c_{\theta}}\right] e_{\omega},
$$

where $\lambda_{\varphi}$ is a positive gain, after the nonlinear term and stability error dynamics are removed and $r_{d}$ is

$$
r_{d}=\left[\dot{\varphi}_{r}-\frac{s_{\phi}}{c_{\theta}} q-\lambda_{\varphi} e_{\varphi}\right] \frac{c_{\phi}}{c_{\theta}} .
$$

According to formula (9), the angular velocity error $e_{\omega}$ is

$$
I \dot{e}_{\omega}=-e_{\omega} I \omega_{B}-e_{\varphi}\left[0,0, \frac{c_{\phi}}{c_{\theta}}\right]^{\mathrm{T}}-A e_{\omega},
$$

where $A \in R^{3 \times 3}$ is a positive gain diagonal matrix.

Similarly, considering the limitation of an orthogonal vector constraint, the dynamics of the direction error under the pseudocontrol of angular velocity $\left[\begin{array}{ll}p_{d} & q_{d}\end{array}\right]^{T}$ is

$$
\dot{e}_{\rho}=-\left(\lambda_{1.1}, \lambda_{1.2}\right) e_{\rho}+Z_{0}(\theta) e_{\omega}
$$

where $Z_{0}(\theta)=\left[Z(\theta) 0_{2 \times 1}\right], \lambda_{1 . i}, k \geq 0(i=1,2)$.

According to the above derivation, the attitude error of the unmanned helicopter represented by the attitude error vector $\left[\begin{array}{lll}e_{\varphi} & e_{\rho} & e_{\omega}\end{array}\right]^{T} \in Z$ is as follows:

$$
\left\{\begin{array}{l}
\dot{e}_{\varphi}=\frac{s_{\phi}}{c_{\theta}} q+\frac{c_{\phi}}{c_{\theta}} r-\varphi_{r} \\
I \dot{e}_{\omega}=-e_{\omega} I \omega_{B}-e_{\varphi}\left[0,0, \frac{c_{\phi}}{c_{\theta}}\right]^{T}-A e_{\omega}, \\
\dot{e}_{\rho}=-\left(\lambda_{1.1}, \lambda_{1.2}\right) e_{\rho}+Z_{0}(\theta) e_{\omega} .
\end{array}\right.
$$

\subsection{Robustness Analysis}

Theorem 1. Suppose that $t \geq t_{0}, \rho_{3,3}(t)$ and expectation $\rho_{d, 3}(t)$ belongs to set $\rho$, then the gain selection is [30]

$$
\left\{\begin{array}{l}
\lambda_{1,1}=\theta_{1}^{2}+k_{1}, \\
\lambda_{1,2}=\eta_{1}^{2}+k_{2}, \\
\lambda_{1,3}=\theta_{1}^{2}+\eta_{1}^{2}+\xi .
\end{array}\right.
$$

In formula (17), $\lambda_{2, \min }$ is the minimum value of the gain matrix. The system attitude error (17) is exponentially stable for the initial condition: $\left[e_{\varphi}(t) e_{\rho}(t) e_{\omega}(t)\right] \in Z$. $\theta_{1}, \theta_{2}, \eta_{1}, \eta_{2}, \xi \geq 0, \theta_{1} \theta_{2} \geq 1 / 2, \eta_{1} \eta_{2} \geq 1 / 2$.

Theorem 2. In the system attitude error (17), given an expected direction vector $\rho_{d}(t)$, when $t \geq t_{0}, \rho_{3,3}(t) \geq 0$, the unmanned helicopter does not turn over and the initial state 


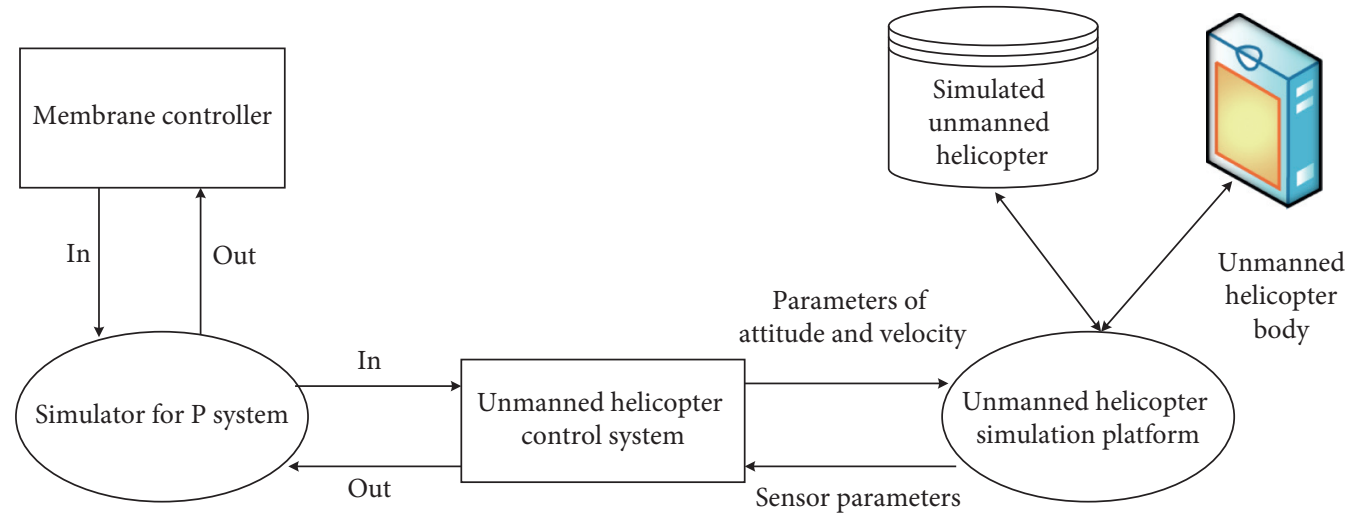

FIGURE 3: Control system of the attitude membrane controller of an unmanned helicopter.

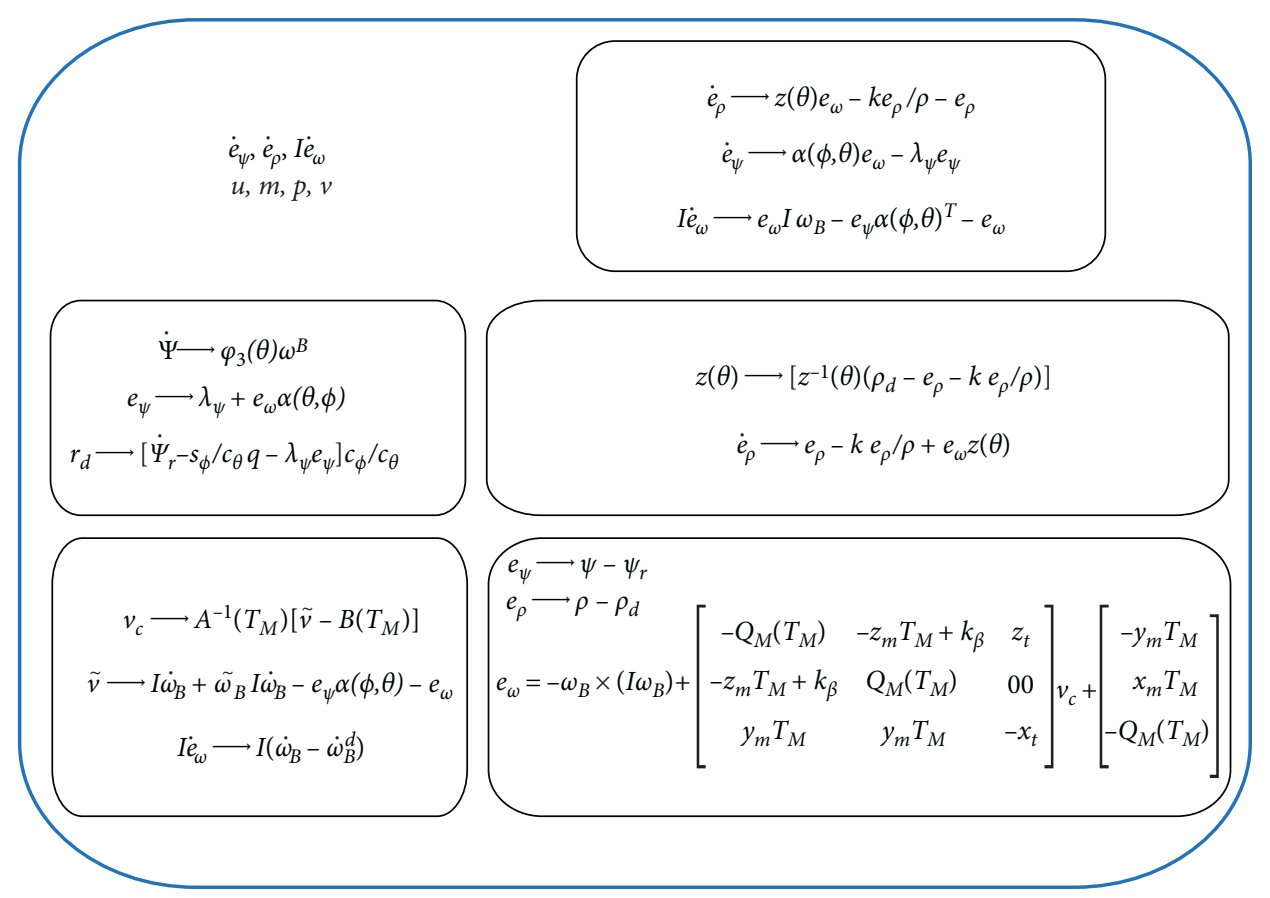

Figure 4: Structure of the attitude membrane controller of an unmanned helicopter.

of the vector to the direction and the angular velocity of the reasoning vector is valid.

Suppose that Theorem 1 is consistent with (17), according to Theorem 1 , the exponential decay of vector $e_{\rho}$ produces inequality:

$$
\left\{\begin{array}{l}
\left\|e_{\rho, 1}\right\| \leq\left\|e_{\rho, 1}\left(t_{0}\right)\right\| e-k_{1}^{\left(t-t_{0}\right)}, \\
\left\|e_{\rho, 2}\right\| \leq\left\|e_{\rho, 2}\left(t_{0}\right)\right\| e-k_{2}^{\left(t-t_{0}\right)}, \\
\forall t \geq t_{0} .
\end{array}\right.
$$

Thereupon, decays the exponent to 0 , and it is bounded:

$$
\left\|e_{\rho, 3}\right\| \leq \frac{2 \sqrt{2}}{\varsigma}\left\|e_{\rho}\left(t_{0}\right)\right\| e^{-k\left(t-t_{0}\right)},
$$

where $\varsigma \in[0,1), k=\min \left(k_{1}, k_{2}\right)$.

\begin{tabular}{|c|c|}
\hline Control parameter & Parameter value \\
\hline$\eta$ & {$\left[\begin{array}{lll}0 & 0 & 0\end{array}\right]$} \\
\hline$k$ & {$\left[\begin{array}{lll}2 & 2 & 0.4\end{array}\right]$} \\
\hline$\xi$ & {$\left[\begin{array}{lll}0.04 & 0.04 & 0.005\end{array}\right]$} \\
\hline$A$ & {$\left[\begin{array}{lll}6 & 6 & 6\end{array}\right]$} \\
\hline$W$ & {$\left[\begin{array}{lll}0.2 & 0.2 & 0.2\end{array}\right]$} \\
\hline$M$ & {$\left[\begin{array}{lll}20 & 8 & 2\end{array}\right]$} \\
\hline$L$ & {$\left[\begin{array}{lll}19 & 6 & 1.5\end{array}\right]$} \\
\hline$\lambda$ & 2 \\
\hline
\end{tabular}

TABle 2: Experimental parameters.

According to Theorem 2, for any $t \geq t_{0}$, there is $\rho_{d, 3} \geq \varsigma, \rho 3,3 \geq 0$, so $\rho_{d, 3}+\rho_{3,3} \geq \varsigma$, that is, $1 / \varsigma \geq 1 / \rho_{d, 3}+\rho_{3,3}$. For $e_{\rho, 3}$, there is 


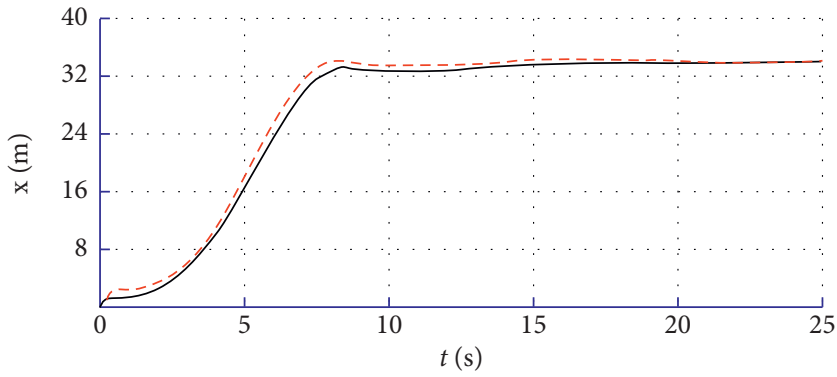

- - - Reference trajectory

- Actual trajectory

(a)

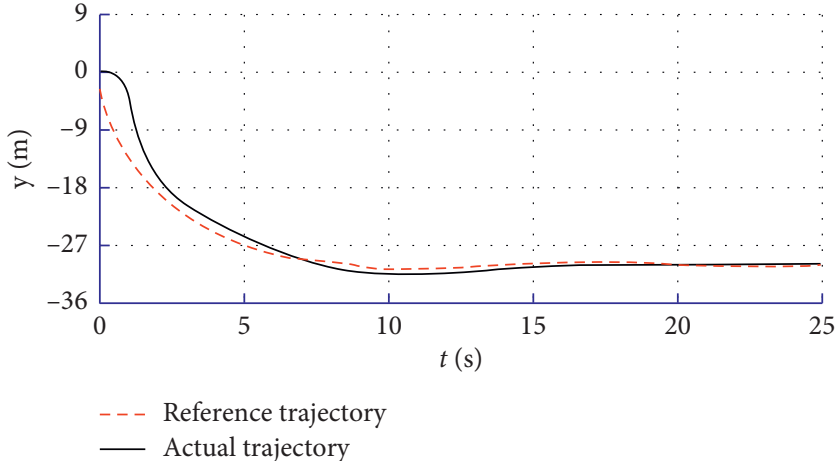

(b)

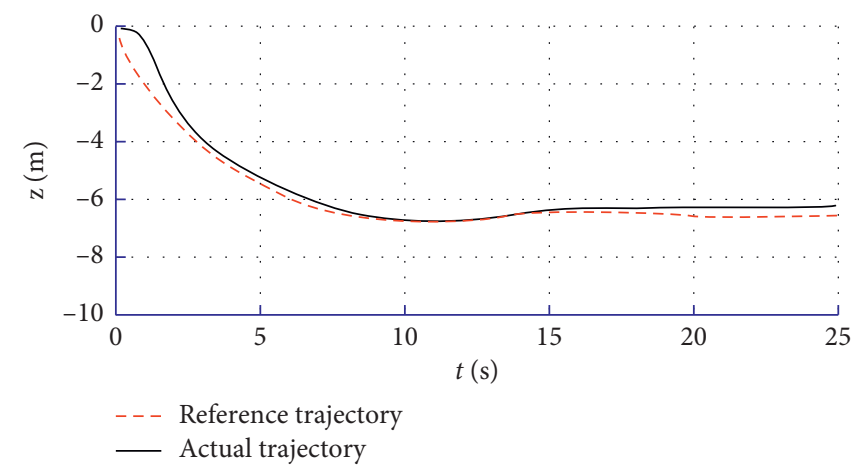

(c)

FIGURE 5: Helicopter trajectory change curve with time.

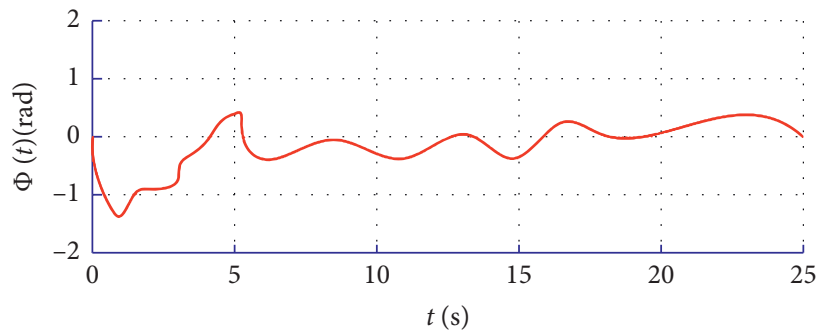

(a)

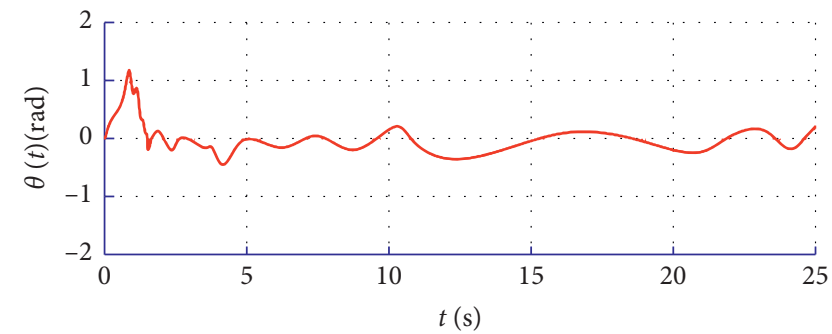

(b)

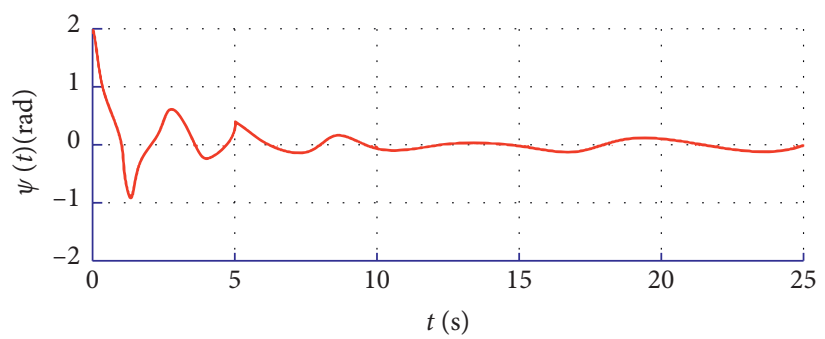

(c)

Figure 6: Helicopter attitude change curve with time. 


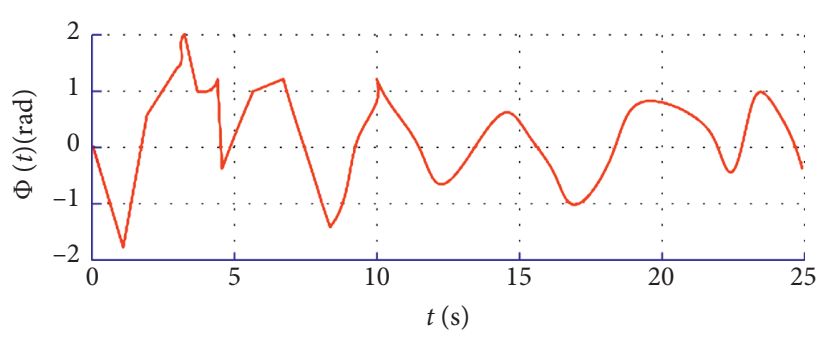

(a)



(b)

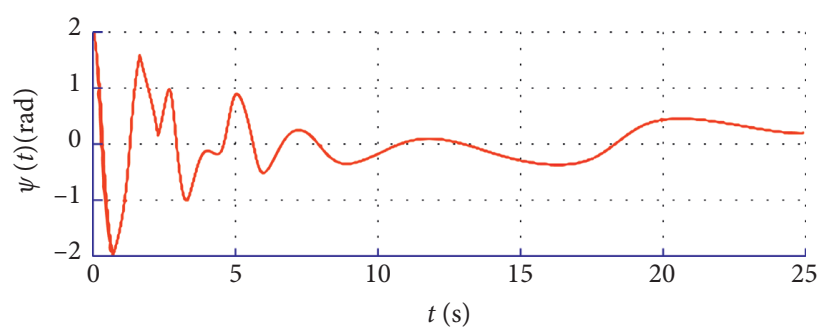

(c)

Figure 7: Time-varying curve of the attitude angle in the disturbed state.

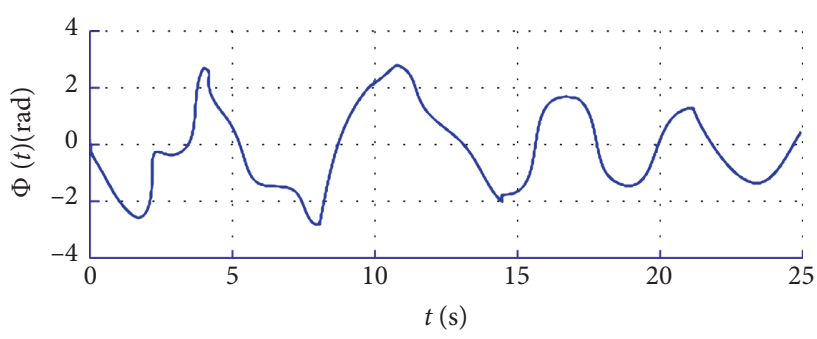

(a)

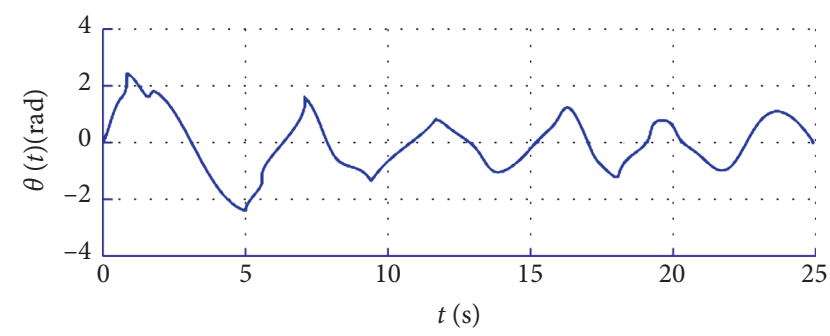

(b)

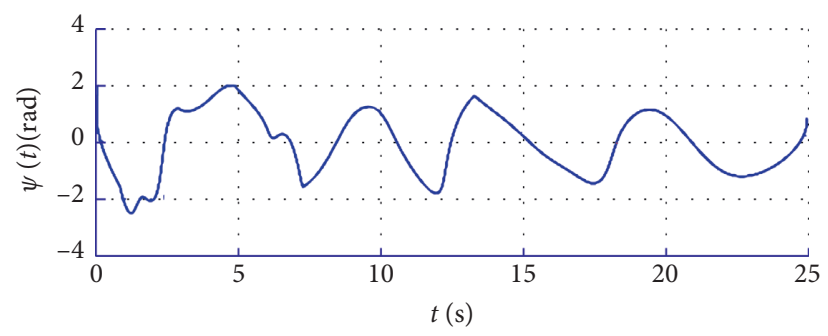

(c)

FIGURE 8: Linear feedback control attitude curve of the helicopter over time.

$$
\begin{aligned}
e_{\rho, 3} & =\rho_{3,3}-\rho_{d, 3} \\
& =\frac{\rho_{d, 1}^{2}+\rho_{d, 2}^{2}-\rho_{3,1}^{2}-\rho_{3,2}^{2}}{\rho_{3,3}+\rho_{d, 3}} \\
& =\frac{-\left(\rho_{3,2}-\rho_{d, 2}\right)\left(\rho_{3,2}+\rho_{d, 2}\right)-\left(\rho_{3,1}+\rho_{d, 1}\right)\left(\rho_{3,1}-\rho_{d, 1}\right)}{\rho_{3,3}+\rho_{d, 3}} \\
& =\frac{-e_{\rho, 2}\left(\rho_{3,2}+\rho_{d, 2}\right)-e_{\rho, 1}\left(\rho_{3,1}+\rho_{d, 1}\right)}{\rho_{3,3}+\rho_{d, 3}},
\end{aligned}
$$

and $e_{\rho, 3}$ norm is

$$
\begin{aligned}
\left\|e_{\rho, 3}\right\| & \leq\left\|\frac{\rho_{3,1}+\rho_{d, 1}}{\rho_{3,3}+\rho_{d, 3}}\right\|\left\|e_{\rho, 3}\right\|+\left\|\frac{\rho_{3,2}+\rho_{d, 2}}{\rho_{3,3}+\rho_{d, 3}}\right\|\left\|e_{\rho, 2}\right\| \\
& \leq \frac{2 \sqrt{2}}{\varsigma}\left\|e_{\rho}\right\| \leq \frac{2 \sqrt{2}}{\varsigma}\left\|e_{\rho}\left(t_{0}\right)\right\| e^{-k\left(t-t_{0}\right)} .
\end{aligned}
$$

Through the above series of analysis and proof, the stability of an attitude error is ensured, and the unmanned helicopter will not turn over in the tracking process and boundary conditions under existence constraints. 


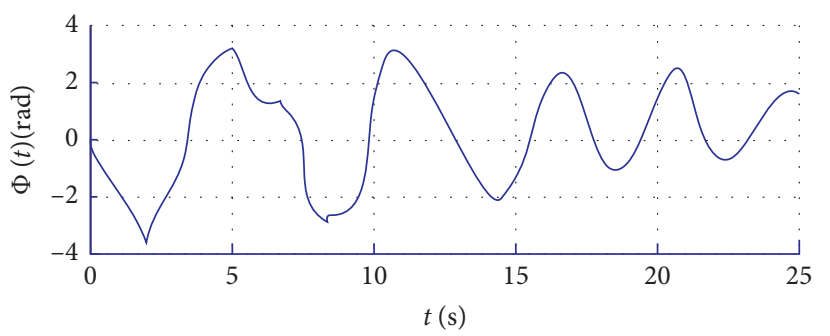

(a)

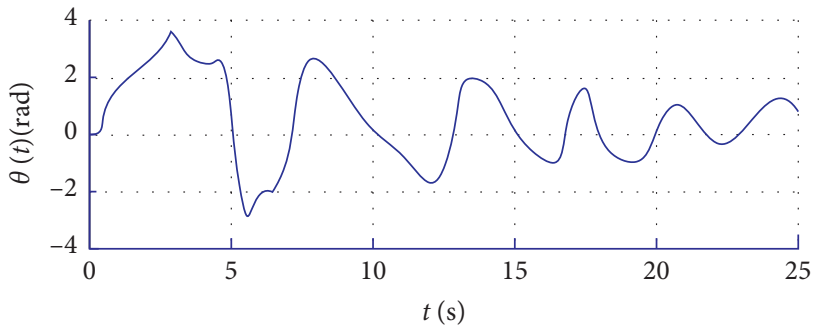

(b)

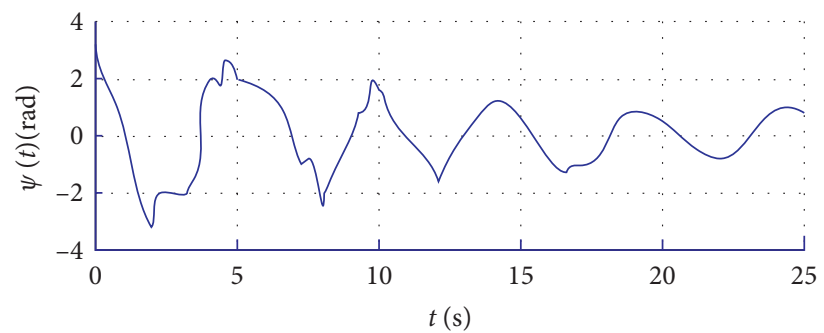

(c)

FIgURE 9: Linear feedback control attitude change curve with time for wind disturbance.

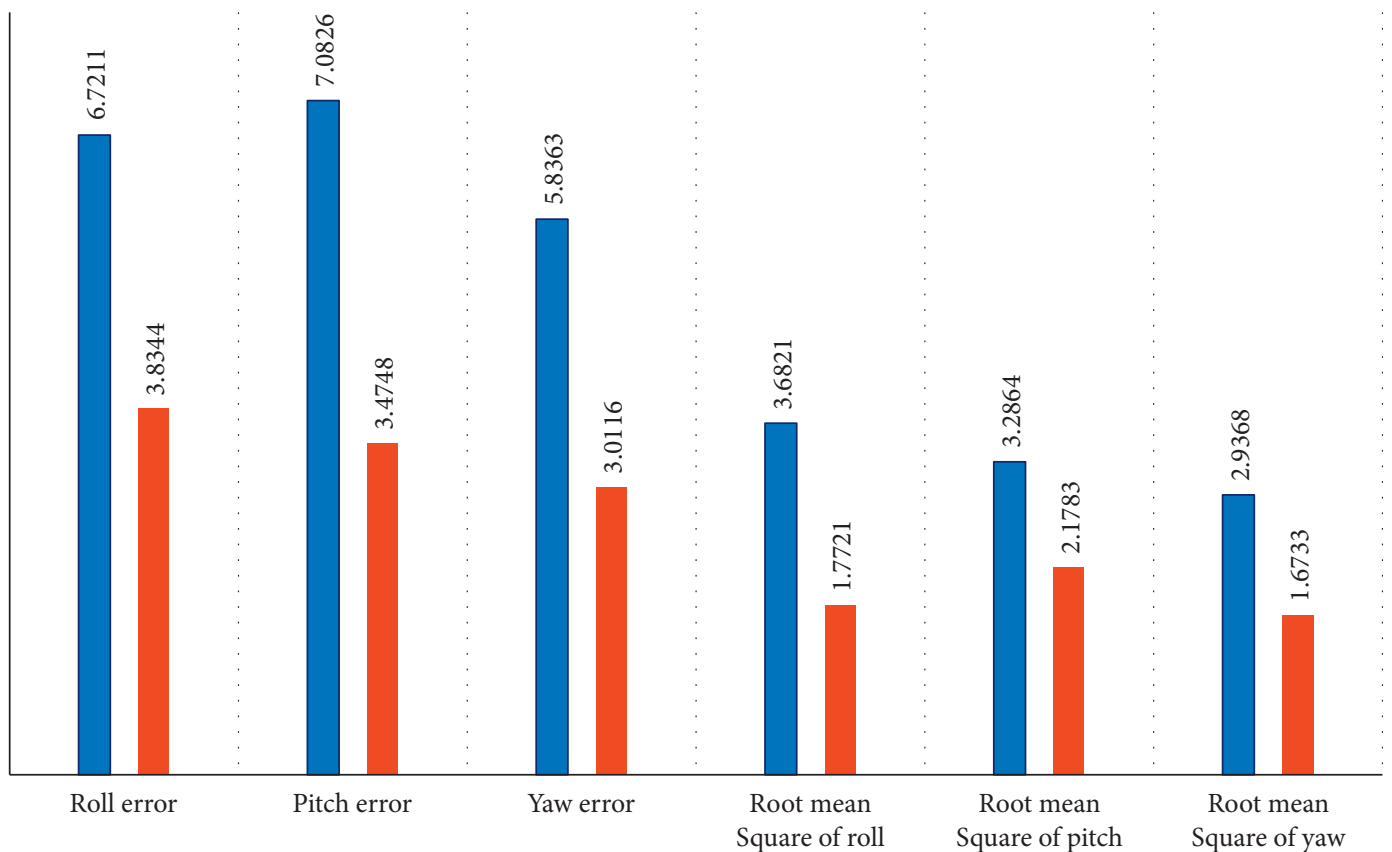

Linear feedback controller

Membrane controller

Figure 10: Experimental error comparison under wind speed disturbance.

\section{Experimental Verification}

4.1. Verification of Attitude Control Performance. To verify the effectiveness of the unmanned helicopter membrane controller designed in this paper in the semiphysical environment, the main hardware parameter information of the experimental platform is shown in Table 1. The software is a membrane system simulator based on the P-Lingua simulation virtual machine, and this software is shown in Figure 3 as a middleware. It can simulate the membrane computer and execute the membrane controller program execution, which facilitates the performance improvement of the membrane simulator and improves computing power of the membrane controller.

The parameters and values of the membrane controller and control gain during the experiment are given in Table 2. The controller gain is adjusted sufficiently according to the 
requirements of Theorem 1 , which further meets the helicopter's need to quickly obtain the desired vector.

The experiment begins by manually manipulating the helicopter to take-off, and after reaching a steady state, the flight state is switched to an automatic flight state. To obtain clear experimental results, the previous $25 \mathrm{~s}$ is selected for the experiment. The trajectory of the position response over time in the inertial coordinate system is shown in Figure 5, and the course of the helicopter channel attitude angle with time is shown in Figure 6.

Figure 5 shows that the membrane controller designed in this paper tends to be stable in about 5 seconds and shows good tracking performance. In Figure 6, the amplitude of the change in the attitude angle with time is controlled in the $\pm 1^{\circ}$ range, and it shows good attitude control performance without interference after the stability.

4.2. Comparison of Attitude Control Performance. The experiment is conducted under the condition of adding wind disturbance. After the helicopter is stable, the wind speed is $10 \mathrm{~m} / \mathrm{s}$ and the disturbance is continued for $25 \mathrm{~s}$. The timevarying curve of the attitude angle in the disturbed state with the membrane controller is shown in Figure 7. Linear feedback control performance experiments are performed in the same environment, and the results of helicopter attitude control performance under normal and wind disturbance are shown in Figures 8 and 9. At the same time, the experimental errors of the linear feedback controller and the membrane controller designed in this paper are compared based on the experimental data, as shown in Figure 10.

It can be seen from the attitude curve of Figure 7 that even if the amplitude of the attitude angle approaches $2^{\circ}$, especially after $5 \mathrm{~s}$, it is relatively stable, which indicates that the membrane controller can control the attitude better under external disturbance. Comparing Figure 6 with Figure 8 , although linear feedback control can also follow the time to control the attitude, the maximum amplitude approaches $2.4^{\circ}$; however, in terms of control performance, the control effect of the membrane controller is obviously higher than that of the linear controller. Similarly, comparing Figure 7 with Figure 9, in the case of wind disturbance, the amplitude of the attitude changes under linear feedback approaching $3.6^{\circ}$. It is obviously higher than $2^{\circ}$ under the membrane controller. Analysis of Figure 10 shows that the attitude control performance of the membrane controller is reduced to approximately 0.4026 in terms of error and root mean square. Through the above comparison and analysis, the control performance of the membrane controller designed in this paper is feasible and has good stability.

\section{Conclusion}

This paper takes the future direction of intelligent and accurate mining as an opportunity, applies an unmanned helicopter to coal mines, fully considers the scenarios of the coal mine environment, realizes model construction and membrane controller design, and through theoretical and experimental verification, it obtains the following conclusions:

(1) It is achievable to construct an unmanned helicopter attitude dynamic model under the complex environment of the coal mines.

(2) It is feasible to realize the attitude membrane controller design of an unmanned helicopter by using membrane computing.

(3) Building the membrane controller is successful, and the unmanned helicopter shows a good control effect under normal or wind disturbance conditions. Through theoretical analysis and comparison with the experimental results of linear feedback control methods, the membrane computing application is further proved for the effectiveness and stability of attitude control of an unmanned helicopter.

The points of the study in this work will provide a new scientific theoretical reference for membrane calculation in the field of optimal control, and at the same time, provide a practical application case for safe and accurate mining of coal mines. Next, we will try to build different membrane computing models, including membrane algorithm design, compared with other control methods, to further optimize the attitude control of an unmanned helicopter and offer new ideas for path planning.

\section{Data Availability}

The experimental data used to support the findings of this study are included within the article.

\section{Conflicts of Interest}

The authors declare that they have no conflicts of interest.

\section{Acknowledgments}

This paper was supported by the National Natural Science Foundation of China (61772033) and the Key Natural Science Research Project of Anhui Higher Education Institutions (KJ2019A0110).

\section{References}

[1] L. Yuan, "Scientific conception of precision coal mining," Journal of China Coal Society, vol. 42, no. 1, pp. 1-7, 2017.

[2] L. Yuan, "Internet of things architecture and key technologies for coal precise mining," Industrial and Mining Automation, vol. 43 , no. 10, 2017.

[3] D. H. Shim, H. J. Kim, and S. Sastry, "Control system design for rotorcraft-based unmanned aerial vehicles using timedomain system identification," in Procedings of the IEEE International Conference on Control Applications IEEE, Anchorage, AK, USA, September 2000.

[4] M. L. Civita, G. Papageorgiou, W. C. Messner, and T. Kanade, "Design and flight testing of a gain-scheduled ho loop shaping controller for a robotic helicopter," in Proceedings of the 2003 American Control Conference, Denver, CO, USA, June 2003. 
[5] A. Schirrer, C. Westermayer, M. Hemedi, and M. Kozek, "LQbased design of the inner loop lateral control for a large flexible BWB-type aircraft," in Proceedings of the 2010 IEEE International Conference on Control Applications, pp. 18501855, IEEE, Yokohama, Japan, September 2010.

[6] H. Liu, G. Lu, and Y. Zhong, "Robust LQR attitude control of a 3-DOF laboratory helicopter for aggressive maneuvers," IEEE Transactions on Industrial Electronics, vol. 60, no. 10, pp. 4627-4636, 2013.

[7] G. V. Lima, R. M. J. Alves de Souza, A. S. de Morais, L. C. O. Lopes, and G. M. V. Ladeira, "Stabilization and path tracking of a mini quadrotor helicopter: experimental results," IEEE Latin America Transactions, vol. 17, no. 3, pp. 485-492, 2019.

[8] A. L'Afflitto, R. B. Anderson, K. Mohammadi, and K. Mohammadi, "An introduction to nonlinear robust control for unmanned quadrotor aircraft: how to design control algorithms for quadrotors using sliding mode control and adaptive control techniques [focus on education]," IEEE Control Systems, vol. 38, no. 3, pp. 102-121, 2018.

[9] T. Oktay, M. Konar, M. Soylak et al., "Increasing performance of autopilot guided small unmanned helicopter," International Journal of Mechanical, Aerospace, Industrial, Mechatronic and Manufacturing Engineering, vol. 10, no. 1, pp. 133-139, 2016.

[10] S. Ozcan, M. U. Salamci, and V. Nalbantoglu, "Multiloop state-dependent nonlinear time-varying sliding mode control of unmanned small-scale helicopter," Proceedings of the Institution of Mechanical Engineers, Part G: Journal of Aerospace Engineering, vol. 234, no. 3, pp. 585-606, 2019.

[11] C. Diao, B. Xian, Q. Yin et al., "A nonlinear adaptive control approach for quadrotor UAVs," in Proceedings of the 2011 8th Asian Control Conference (ASCC), IEEE, Kaohsiung, Taiwan, pp. 223-228, May 2011.

[12] X. Y. Sun, Y. C. Fang, and N. Sun, "Backstepping-based adaptive attitude and height control of a small-scale unmanned helicopter," Control Theory \& Applications, vol. 29, no. 3, pp. 111-118, 2012.

[13] B. Ahmed and H. R. Pota, "Flight control of a Rotary wing UAV using adaptive backstepping," International Journal of Robust \& Nonlinear Control, vol. 20, no. 6, pp. 639-658, 2010.

[14] I. Ullah and H.-L. Pei, "Sliding mode tracking control for unmanned helicopter using extended disturbance observer," Archives of Control Sciences, vol. 29, no. 1, pp. 169-199, 2019.

[15] D. Nodland, H. Zargarzadeh, and S. Jagannathan, "Neural network-based optimal adaptive output feedback control of a helicopter UAV," IEEE Transactions on Neural Networks and Learning Systems, vol. 24, no. 7, pp. 1061-1073, 2013.

[16] D. J. Lee and H. Bang, "Reinforcement learning based neurocontrol systems for an unmanned helicopter," in Proceedings of the ICCAS 2010, IEEE, Gyeonggi-do, South Korea, pp. 2537-2540, October 2010.

[17] A. N. Hang and X. Bin, "Attitude reinforcement learning control of an unmanned helicopter with verification," Control Theory \& Applications, vol. 36, no. 4, pp. 516-524, 2019.

[18] T. Oktay and C. Sultan, "Constrained predictive control of helicopters," Aircraft Engineering and Aerospace Technology, vol. 85, no. 1, pp. 32-47, 2013.

[19] M. Hossein Khalesi, H. Salarieh, and M. Saadat Foumani, "System identification and robust attitude control of an unmanned helicopter using novel low-cost flight control system," Proceedings of the Institution of Mechanical Engineers, Part I: Journal of Systems and Control Engineering, vol. 234, Issue 5, 2020.
[20] Q. Wang and Q. Zhao, "Rotor aerodynamic shape design for improving performance of an unmanned helicopter," Aerospace Science and Technology, vol. 87, pp. 478-487, 2019.

[21] T. Oktay and F. Sal, "Combined passive and active helicopter main rotor morphing for helicopter energy save," Journal of the Brazilian Society of Mechanical Sciences and Engineering, vol. 38, no. 6, pp. 1511-1525, 2016.

[22] A. Belkadi, Z. Liu, L. Ciarletta, Y. Zhang, and D. Theilliol, "Flocking control of a fleet of unmanned aerial vehicles," Control Theory and Technology, vol. 16, no. 2, pp. 82-92, 2018.

[23] I. A. Raptis and K. P. Valavanis, Linear and Nonlinear Control of Small-Scale Unmanned helicopters, Springer Science \& Business Media, Berlin, Germany, 2010.

[24] G. Păun, "Computing with membranes," Journal of Computer and System Sciences, vol. 61, no. 1, pp. 108-143, 2000.

[25] G. Zhang, M. Gheorghe, L. Pan, and M. J. Pérez-Jiménez, "Evolutionary membrane computing: a comprehensive survey and new results," Information Sciences, vol. 279, pp. 528-551, 2014.

[26] B. Yun, Research on Posture Control Method of Coal Mine Snake Detection Robot, Xi'an University of Science and Technology, Xi'an, China, 2019.

[27] I. A. Raptis and K. P. Valavanis, Linear and Nonlinear Control of Small-Scale Unmanned Helicopters, Springer, Berlin, Germany, 2011.

[28] N. A. V. Piercy, Aerodynamics, English Universities Press, USA, 1947.

[29] G. Păun and R. Păun, "Membrane computing and economics: numerical P systems," Fundamenta Informaticae, vol. 73, no. 1-2, pp. 213-227, 2006.

[30] B. Godbolt and A. F. Lynch, "An unmanned helicopter control with partial small body force compensation: experimental results," Robotica, vol. 36, no. 10, pp. 1436-1453, 2018. 